\title{
Positronium annihilation in high-purity liquids
}

\author{
S.J. WANG, Y.C. JEAN* and H. ZHANG* \\ Department of Physics, Wuhan University, Wuhan, China \\ * University of Missouri-Kansas City, Kansas City, MO 64110, U.S.A.
}

\begin{abstract}
The positron lifetime measurements have been performed as a function of temperature for liquid methane and propane and as a function of magnetic field for liquid argon. The long o-Ps lifetime, high o-Ps intensity, and a strong temperature dependence of these values are observed, and interpreted as being due to the Ps localization in the bubble state. The bubble size and surface tension are evaluated using bubble model and o-Ps lifetime. The chemical reaction of Ps atom with oxygen impurities was studied. The reaction rate $\mathrm{K}_{\mathrm{ps}}$ and its temperature behavior in liquid methane and propane were obtained. The results of magnetic field experiments in both pure and oxygen-added liquid argon show that the lifetime of $\mathrm{o}$-Ps with magnetic quantum number $\mathrm{m}=0$ state decreases basically according to the normal Zeeman effect.
\end{abstract}

\section{Introduction}

In recent years, there has been a growing interest in the study of the behavior of Positronium (Ps), the bound state of a positron and an electron, in liquid system ${ }^{[1,2]}$. The Ps atom is found to be localized in a microscopic cavity, called a Ps bubble state. The bubble model was first suggested by Ferrell ${ }^{[3]}$ to explain an unexpectedly long o-Ps lifetime in liquid helium. The bubble radius was determined by a balance between the outward pressure caused by the Ps zero-point motion and the shrinking forces due to surface tension and external pressure. In the presence of a chemically reactive species, the o-Ps lifetime is significantly reduced by a process called chemical quenching. The strength of chemical quenching depends on the chemical and physical properties of the molecules. The application of static magnetic field causes a mixing of $\mathrm{p}$-Ps and the o-Ps with $\mathrm{m}=0$ states and changes their annihilation parameters. Along this line of work, the liquid Ar is a special system. In absence of magnetic field, the p-Ps intensity measured by ACAR is not equal to $1 / 3$ of o-Ps intensity existing reported by positron lifetime experiments ${ }^{[1]}$. This discrepancy remains unresolved for many years. For magnetic quenching experiments, the ACAR results show an unexpected small increase of the narrow component ${ }^{[4]}$. In this paper, we report the results of positron lifetime measurements in high purity liquid $\mathrm{CH}_{4}, \mathrm{C}_{3} \mathrm{H}_{8}$ and $\mathrm{Ar}$ to study the Ps bubble state, chemical reaction, and magnetic quenching. 


\section{Experiments}

The research grade gas $\left(\mathrm{CH}_{4}, \mathrm{C}_{3} \mathrm{H}_{8}\right.$ or $\left.\mathrm{Ar}\right)$ was first passed through a purifier to reduce oxygen and other impurities to less than $1 \mathrm{ppm}$ and then condensed to solid $\left(\mathrm{CH}_{4}\right.$ and $\mathrm{C}_{3} \mathrm{H}_{8}$ ) or liquid ( $\mathrm{Ar}$ ) in a sample cell made of aluminum which was mounted on a cool head of closed-cycle He refrigerator with a temperature control better than $\pm 0.1 \mathrm{~K}$ over a range from 10 to $300 \mathrm{~K}$. For oxygen quenching experiments, the oxygen and pure gas were first mixed in the gas line and then condensed. $\mathrm{A}^{22} \mathrm{Na}$ positron source, which sealed between two thin Al foils $(6 \mu \mathrm{m})$ was used in the liquid experiments. The positron lifetime measurements were performed using conventional fast-fast coincident system. For magnetic quenching, the scintillators and magnetically shield photomultiplier were separated by lucite light pipes $(18 \mathrm{inch})$ in order to eliminate the effect of magnetic field. The time resolution of the system was 280 ps for temperature experiments and 400 ps for magnetic quenching.

We measured the positron lifetime spectra as a function of temperature for liquid $\mathrm{CH}_{4}$ and $\mathrm{C}_{3} \mathrm{H}_{8}$, and as a function of magnetic field ranging from 0 to $2.0 \mathrm{~T}$ for liquid Ar. The lifetime spectra were analyzed using the PATFIT computer program ${ }^{[5]}$. In absence of magnetic field, three lifetime components were resolved, and the $\tau_{1}, \tau_{2}, \tau_{3}$ are assigned to $\mathrm{p}$-Ps, positron and o-Ps annihilation ,respectively. For liquid $\mathrm{Ar}$, in the presence of magnetic field, the spectra were analyzed by four components by constraining the longest lifetime $\tau_{4}=\tau_{3}$ of liquid $\mathrm{Ar}$ at zero field due to the annihilation of o-Ps with $\mathrm{m}= \pm 1$ and $\mathrm{I}_{3} / \mathrm{I}_{4}=1 / 2$ according to the population of ground states of o-Ps. A source correction was made in data analysis.

\section{Results and Discussions}

\section{Ps-bubble State}

Positron lifetime measurement has been provided a wealth of useful information concerning bubble formation in high purity liquid. A sharp transition from solid to liquid was observed ${ }^{[6]}$, i.e., from 2.3 to $4.8 \mathrm{~ns}, 1.2$ to $2.9 \mathrm{~ns}$ and 2.4 to $5.0 \mathrm{~ns}$ for high purity liquid $\mathrm{CH}_{4}, \mathrm{C}_{3} \mathrm{H}_{8}$ and $\mathrm{Ar}$, respectively. The results indicated that the annihilation sites of Ps in liquid are different from that in solid. The Ps is believed to localized in bubble state which is a result of strong exchange repulsion between the electron of Ps and the tightly bound electron in the medium.

The temperature dependencies of o-Ps lifetime and intensity of liquid $\mathrm{CH}_{4}$ and $\mathrm{C}_{3} \mathrm{H}_{8}$ were shown by solid cycles in Fig.1-2, and Fig.3-4, respectively. From Fig.1 and Fig.3, the o-Ps lifetimes increase with the increasing temperature, i.e. from 4.8 to $6.4 \mathrm{~ns}$ for $\mathrm{CH}_{4}$ and from 2.9 to $3.8 \mathrm{~ns}$ for $\mathrm{C}_{3} \mathrm{H}_{3}$. However, the variations of o-Ps intensities shown in Fig. 2 and Fig. 4 have different trend, i.e. while the temperature increased, the o-Ps intensity of liquid $\mathrm{CH}_{4}$ is nearly constant, but a slight increase of o-Ps intensity was observed for liquid $\mathrm{C}_{3} \mathrm{H}_{8}$. The reason may be the different physical properties in liquids $\mathrm{CH}_{4}$ and $\mathrm{C}_{3} \mathrm{H}_{8}$. According to the $\mathrm{Ps}$ bubble model and measured o-Ps lifetime, the surface tension and bubble size in liquid $\mathrm{CH}_{4}$ are evaluated and shown in Table 1 along with other physical parameters ${ }^{[6]}$.

Finally, we have measured positron lifetime spectra in liquid $\mathrm{Ar}$ at $86 \mathrm{~K}$ and solid $\mathrm{Ar}$ at $60 \mathrm{~K}$. In solid Ar, we observed a $I_{3}$ of $8.77 \%$ with a $\tau_{3}=2.38 \mathrm{~ns}$, which agree with the existing reported results $[7,8]$. However, in liquid Ar, we observed a large value of $I_{3}=24.48 \%$, which agrees with the estimated value of $\mathrm{I}_{3}=22 \pm 4 \%$ by ACAR measurement ${ }^{[1]}$, while the existing lifetime results show only in the order of $10 \%$. The large difference of o-Ps intensity between the present work and the existing results is thought due to the presence of impurity in the Ar sample. 


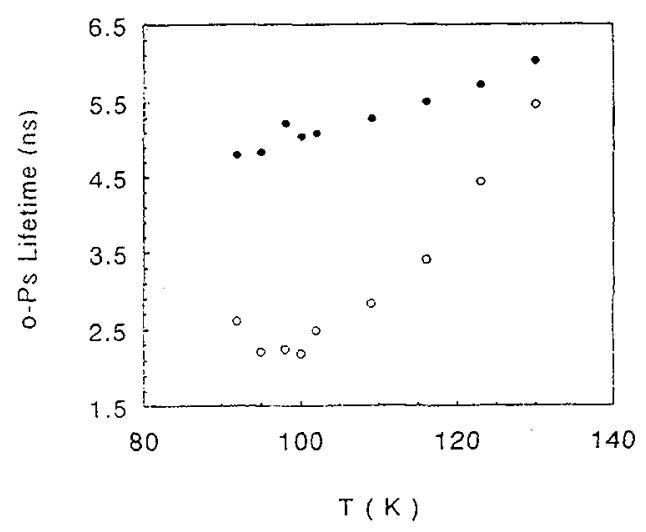

Fig. 1 The variations of o-Ps lifetime with temperature in pure (†) and $\mathrm{O}_{2}$-added $(0)$ liquid $\mathrm{CH}_{4}$

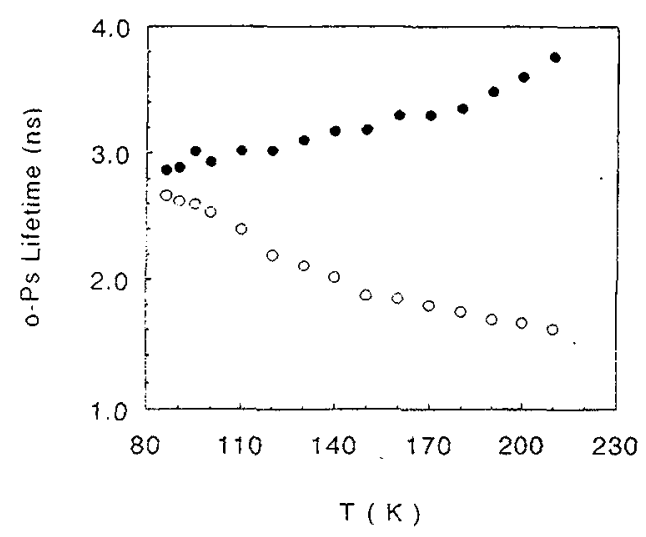

Fig. 3 The variations of o-Ps lifetime with temperature in pure (1) and $\mathrm{O}_{2}$-added (O) liquid $\mathrm{C}_{3} \mathrm{H}_{8}$



Fig.2 The variations of o-Ps intensity with temperature in pure (๑) and $\mathrm{O}_{2}$-added (o) liquid $\mathrm{CH}_{4}$

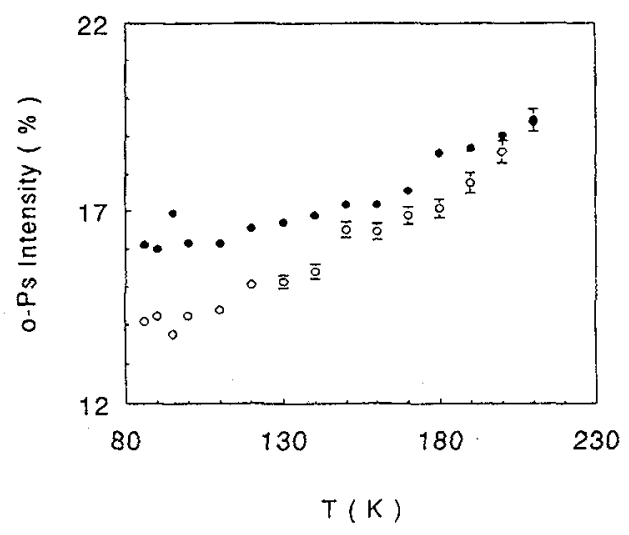

Fig. 4 The variations of o-Ps intengity with temperature in pure ( and $\mathrm{O}_{3}$-added (0) liquid $\mathrm{C}_{3} \mathrm{H}_{8}$ 
Table 1. The bubble radius $(\mathrm{R})$ and surface tension $(\gamma)$ for liquid methane

\begin{tabular}{rrrrrr}
\hline $\mathrm{T}(\mathrm{K})$ & $\tau_{3}(\mathrm{~ns})$ & $\begin{array}{c}\text { Density } \\
\left(\mathrm{gcm}^{-3}\right)\end{array}$ & $\begin{array}{c}\text { Pressure } \\
(\text { Torr })\end{array}$ & $\mathrm{R}(\AA)$ & $\gamma\left(\mathrm{dyn} \mathrm{cm}^{-1}\right)$ \\
\hline 93 & $4.85 \pm 0.04$ & 0.4499 & 350 & 4.67 & 18.0 \\
95 & $4.94 \pm 0.04$ & 0.4472 & 465 & 4.71 & 17.6 \\
102 & $5.09 \pm 0.04$ & 0.4378 & 517 & 4.78 & 16.3 \\
109 & $5.29 \pm 0.04$ & 0.4280 & 724 & 4.87 & 15.1 \\
116 & $5.57 \pm 0.04$ & 0.4180 & 1134 & 5.00 & 13.8 \\
123 & $5.73 \pm 0.03$ & 0.4077 & 1758 & 5.07 & 12.5 \\
131 & $6.04 \pm 0.04$ & 0.3951 & 2895 & 5.19 & 11.0 \\
140 & $6.56 \pm 0.04$ & 0.3797 & 4705 & 5.40 & 9.4 \\
150 & $7.28 \pm 0.04$ & 0.3611 & 7497 & 5.67 & 7.5 \\
\hline
\end{tabular}

2.Chemical Reaction with Oxygen

Oxygen is known as an effective chemical quencher. We measured the positron lifetime spectra as a function of temperature for liquid $\mathrm{CH}_{4}$ and $\mathrm{C}_{3} \mathrm{H}_{8}$ containing $6.875 \times 10^{-2} \mathrm{Mol}$ and $6.0 \times 10^{-2} \mathrm{Mol}$ oxygen, respectively. The results of o-Ps annihilation parameters are also shown in Fig. 1-4 by the cycles. We found that the effects of oxygen quenching on o-Ps annihilation parameter are quite different for liquid $\mathrm{CH}_{4}$ and $\mathrm{C}_{3} \mathrm{H}_{8}$. Furthermore, the reaction rate $\mathrm{K}_{\mathrm{ps}}$ can be evaluated using the following equation ${ }^{[2]}$

$$
\lambda_{3}=\lambda_{3}{ }_{3}+\mathrm{K}_{\mathrm{ps}}[\mathrm{M}]
$$

where [M] is the molar concentration of oxygen, and $\lambda_{3}{ }_{3}$ and $\lambda_{3}$ are o-Ps annihilation rates in pure and in oxygen-added liquid respectively. One of the most fascinating observations in Ps chemistry is the variation of $\mathrm{K}_{\mathrm{ps}}$ with temperature ${ }^{[9]}$. We plot the $\ln \left(\mathrm{K}_{\mathrm{ps}}\right)$ against $1 / \mathrm{T}$ in Fig.5-6 for liquid $\mathrm{CH}_{4}$ and $\mathrm{C}_{3} \mathrm{H}_{8}$. For liquid $\mathrm{CH}_{4}$, the $\mathrm{K}_{\mathrm{ps}}$ increases with temperature at low temperature, and reaches a maximum value at $\mathrm{T}_{0}(100 \mathrm{~K})$, the so-called turnover temperature, then decreases above $\mathrm{T}>100 \mathrm{~K}$. A normal Arthenius behavior at $\mathrm{T}<100 \mathrm{~K}$ and opposite that at $\mathrm{T}>100 \mathrm{~K}$ were observed in Fig.5. So the "normal" and "negative" activation energies $E_{2}=3.70 \mathrm{KJ} / \mathrm{Mol}$ and $E_{2}^{\prime}=-8.68 \mathrm{KJ} / \mathrm{Mol}$ were obtained from least square fitting of the right and left portions of the curve. For liquid $\mathrm{C}_{3} \mathrm{H}_{8}$, in the temperature range of $85-210$ $\mathrm{K}$, we only observed the increase of $\mathrm{K}_{\mathrm{ps}}$ with the increasing temperature, and the normal activation energy $\mathrm{E}_{\mathrm{a}}=3.28 \mathrm{KJ} / \mathrm{Mol}$ was evaluated.

\section{Magnetic Quenching}

Theoretical aspects of magnetic quenching of Ps annihilation have been developed by Halpern and by Mills ${ }^{[10,11]}$. The application of magnetic field has no effect on positron nor on o-Ps with $m= \pm 1$ states, but has an effect on $\mathrm{p}-\mathrm{Ps}$ and o-Ps with $\mathrm{m}=0$ states. The annihilation rates $\lambda_{1}(\mathrm{H})$ and $\lambda_{3}(\mathrm{H})$ for the mixed $\mathrm{p}$-Ps and o-Ps with $\mathrm{m}=0$ states as a function of external magnetic field $\mathrm{H}$ follow ${ }^{[12]}$ :

$$
\lambda_{1}(H)=\left(\lambda_{1}(0)+\mathrm{a}^{2} \lambda_{3}(0)\right) /\left(1+\mathrm{a}^{2}\right)
$$




\section{$\mathrm{O}_{2} \ln$ Liquid $\mathrm{CH}_{4}$}

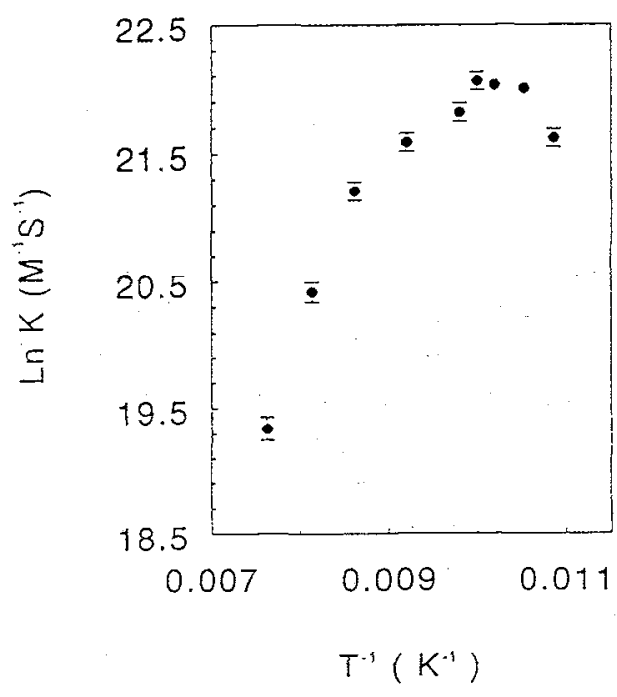

Fig.5. The plot of $\ln \left(\mathrm{K}_{\mathrm{fg}}\right)$ vs. $1 / \mathrm{T}$ in chemical reaction for PS with oxygen in liquid $\mathrm{CH}_{4}$

Liquid Ar (86K)

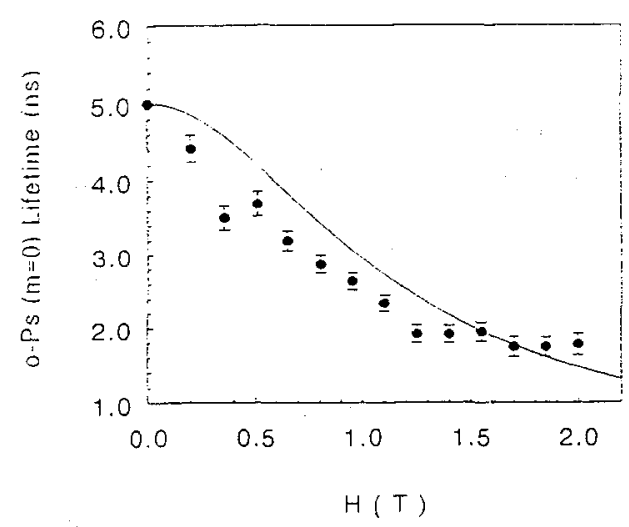

Fig.7 The lifetime of $0-P s$ with $m=0$ in liquid Ar at $86 \mathrm{~K}$ as a function of magnetic field. The solid line is theoretical value for $n=1$
$\mathrm{O}_{2}$ In Liquid $\mathrm{C}_{3} \mathrm{H}_{8}$

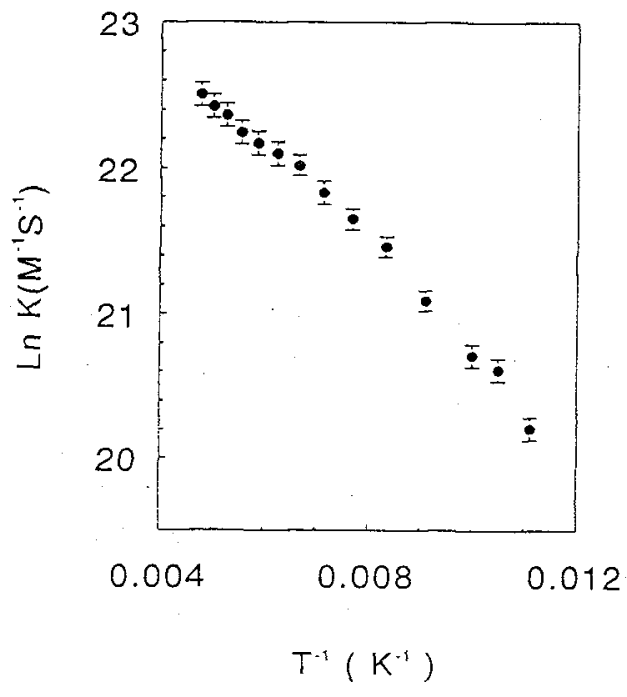

Fig.6 The plot of $\ln \left(\mathrm{K}_{\mathrm{ps}}\right)$ vs. $1 / \mathrm{T}$ in chemical reaction for Ps with oxygen in liquid $\mathrm{C}_{3} \mathrm{H}_{8}$

Liquid Ar + Oxygen (86K)

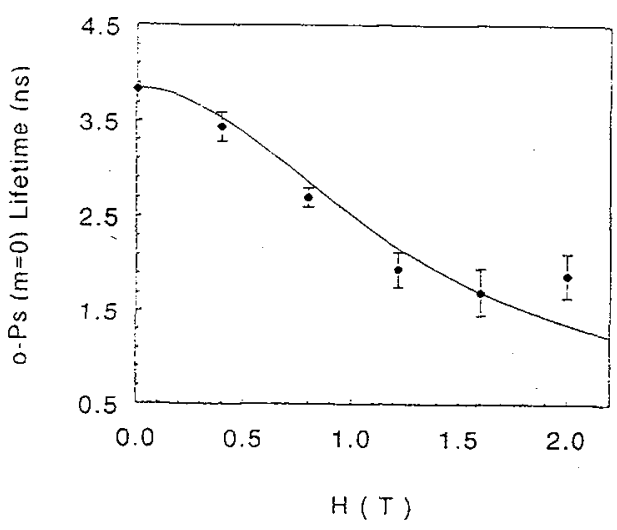

Fig.8 The lifetime of $0-\mathrm{Ps}$ with $\mathrm{m}=0$ in $\mathrm{O}_{2}$-added liquid $\mathrm{Ar}$ at $86 \mathrm{~K}$ as a function of magnetic field. The solid line is theoretical value for $\eta=1$ 


$$
\lambda_{3}(\mathrm{H})=\left(\lambda_{3}(0)+\mathrm{a}^{2} \lambda_{1}(0)\right) /\left(1+\mathrm{a}^{2}\right)
$$

with

$$
\begin{aligned}
& a=\left(\sqrt{1+x^{2}}-1\right) / x \\
& x=4 \mu_{B} H / \Delta E \\
& \Delta E=\eta \Delta E_{v a c}
\end{aligned}
$$

where $\mu_{\mathrm{B}}$ is the Bohr magneton, $\eta$ is used to describe the effect of medium on the Ps wavefunction and very close 1 for the $P s$ trapped in a bubble state, $\Delta \mathrm{E}$ is the hyperfine splitting and $\Delta \mathrm{E}_{\mathrm{vac}}\left(=8.45 \times 10^{-4} \mathrm{eV}\right)$ is the vacuum value of $\Delta \mathrm{E}$. Consequently, the measured lifetime spectra contain two distinct o-Ps components contributed from o-Ps with $\mathrm{m}=0$, and from o-Ps with $\mathrm{m}= \pm 1$ states, respectively. The lifetime of $o$-Ps with $m=0$ decreases as a function of magnetic field while the lifetime with $m= \pm 1$ states is unchanged. In four-lifetime analysis, they are designated as $\tau_{3}$ and $\tau_{4}$, for $\mathrm{m}=0$ and $\mathrm{m}= \pm 1$, respectively. The $\tau_{1}$ increases slightly but $\tau_{3}$ decreases largely with magnetic field according to eqs.(2) and (3), respectively. Since $\tau_{1}$ only slightly increases from 0.122 to $0.130 \mathrm{~ns}$ for the magnetic field ranging from 0 to $2.0 \mathrm{~T}$. So we analyzed all lifetime spectra with a fixed value of $\tau_{1}=0.125 \mathrm{~ns}$. The results of $\tau_{3}$, the lifetime of o-Ps with $\mathrm{m}=0$ state, as a function of magnetic field are plotted in Fig.7. As expected, $\tau_{3}$ decreases with increasing of the field. The variation of $\tau_{3}$ basically follows the theoretical value plotted by solid line for $\eta=1$. As expected we observe nearly constant values of $\tau_{2}$ and $I_{2}$. The sum of o-Ps intensities (i.e. $\mathrm{I}_{3}+\mathrm{I}_{4}$ ) is also nearly constant value indicates that the magnetic field has no effect on the Ps formation mechanism, at least up to $2.0 \mathrm{~T}$. In oxygen-added liquid Ar, the lifetimes $\tau_{3}$ of o-Ps with $\mathrm{m}=0$ state vs $\mathrm{H}$ are shown in Fig. 8 as a function of magnetic field. The variation of $\tau_{3}$ follows the theoretical prediction for $\eta=1$. These results indicate the lifetime of $o-P s$ with $m=0$ state decreases basically according to the normal Zeeman effect.

\section{REFERENCES}

1. Stewart,A.T., Briscoe,C.V., and Steinbacher,J.J., Can. J. Phys., 68(1990)1362

2. Schrader,D.M. and Jean,Y.C., eds., "Positron and Positroniun Chemistry" (Elsevier, Amsterdam, 1988)

3. Ferrell,R.A., Phys. Rev., 108(1957)167

4. Steinbacher,J.J. and Stewart,A.T., Mat. Sci. Forum, 105-110(1992)1711

5. Kirkegaard,P., Eldrup,M., Mogensen,O.E. and Pedersen,N.J., Comp. Phys. Comm., 23, (1981) 307

6. Wang,S.J., Nakanishi,H. and Jean,Y.C., J. Phys.:Condens. Matter, 3,(1991)2167

7. Jean, Y.C. Yu, C., Zhou, D.M. Phys. Rev. B., 32 (1985) 4313

8. Rytsola, K., Vettenranta, J., Hautojarvi,P., J.Phys. B. 17 (1984) 3359

9. Nakanishi,H., Wang,S.J. and Jean,Y.C.,in "Positron Annihilation", edited by L.DorikensVanpraet et al.(World Sci. Singapore,1988),p740

10. Halpern,O., Phys. Rev., 94(1954)904

11. Mills, Jr., A.P., J.Chem. Phys. 62 (1975) 2647

12. Dupasquier,A., De Natale,P. and A. Rolando, Phys. Rev. A, 43(1991)10036 